\title{
Synchrotron light through ancient glass
}

\section{De Raedt, B. Vekemans, K. Janssens, F. Adams}

\begin{abstract}
Synchrotron Radiation induced micro $S_{\text {X-ray Fluorescence ( } \mu \text {-SRXRF) can }}$ provide complementary information on trace elements when used together with EPMA (Electron Probe Micro Analysis). EPMA has a limited sensitivity with detection limits of $0.1 \%$ in the most favourable conditions. Offering ppm level detectability for many more elements, SRXRF is considerably more powerful for quantitative fingerprint analysis in order to gain a better understanding of the provenance of archaeological objects. In this paper, after a short description of the $\mu$-SRXRF instrumentation, a study on sixteenth and seventeenth century glass objects from Antwerp will be briefly outlined.
\end{abstract}

\section{Instrumentation}

The $\mu$-SRXRF measurements were executed at the Hasylab (Hamburger Synchrotron Labor, Hamburg, Germany) beamline $\mathrm{L}$ using a $20 \times 20 \mu \mathrm{m}^{2}$ white synchrotron X-ray beam. A photograph and schematic drawing of the experimental set-up is shown in Figure 1. Quantification of the $\mu$-SRXRF spectra was performed using a fundamental parameter method [1]. The accuracy of the method was checked as with the EPXMA measurements by means of a series of glass standards with certified trace composition [2].

\section{Analysis of sixteenth and seventeenth century Antwerp glass vessels}

Archaeological excavations in the historical center of Antwerp have yielded an important number of sixteenth and seventeenth century glass made 'à la façon-deVenise' (Figure 2). The basic assumption is that most of these objects are locally manufactured, on the other hand some of them may have been imported from

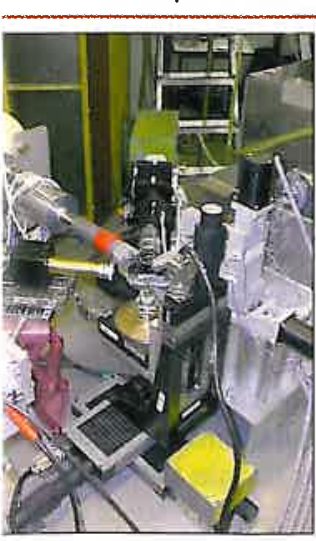

Venice. With the aim of making a distinction between local and Venetian production, the major and trace composition of about 130 soda-lime glass vessels was determined.

Figure 3(a), a binary plot of the amount of $\mathrm{K}_{2} \mathrm{O}$ versus the amount of $\mathrm{CaO}$, reveals the existence of four distinct compositional groups. Two groups, labeled 'Antwerp cristallo' and 'Antwerp vitrum blanchum', show major compositions nearly identical to the composition of two genuine Venetian glass types, 'cristallo' and 'vitrum blanchum' [3], suggesting that these objects might have been imported. The two other categories, named 'Antwerp façonde-Venise' and 'Antwerp mixed alkali', appear to be specific for the Antwerp production. It is however surprising that the proportion of the cristallo and vitrum blanchum glass appears to be important among the Antwerp finds. In previous studies on glass from London and Amsterdam of the same period it seemed that only a few vitrum blanchum glasses are found and glass with the 'cristallo' composition occurred even more rarely $[4,5]$. In order to investigate whether indeed the cristallo glass and vitrum blanchum glass was imported and made with the same raw materials as genuine Venetian glass vessels, i.e. very pure quartz pebbles and purified Levantine soda ashes, the trace element composition was determined. In addition, a number of glass vessels from Venice were analyzed as reference [3]. The study of the trace element content revealed that the amount of $\mathrm{Zr}$ was especially distinctive for differentiating local production from Venetian import. Figure 3(b) shows a binary plot of the amount of $\mathrm{Zr}$ versus the amount of $\mathrm{Sr}$. Whereas the majority of the Antwerp finds features a $\mathrm{Zr}$ content between 40 and $100 \mathrm{ppm}$, the truly Venetian fragments and a limited number of Antwerp vessels show a significantly lower $\mathrm{Zr}$ concentration of $10-20$ ppm.

Fig 1: (left) a photograph of the $\mu-$ SRXRF spectrometer installed at beam line L, Hasylab with C: capillary unit, D: detector, M: microscope and S: sample holder on $X Y Z$ motor stage; (top) a schematic drawing of the SRXRF spectrometer

\section{Conclusions}

This work illustrates the complementary nature of EPMA and $\mu$-SRXRF. The major and trace composi-

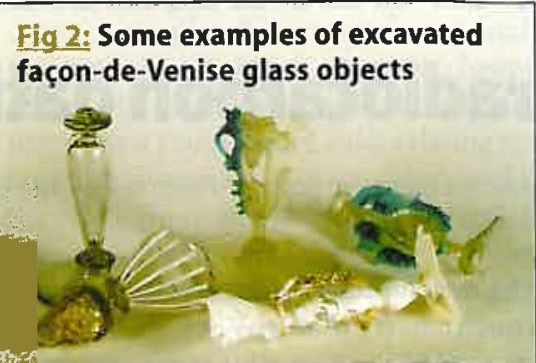

(a)

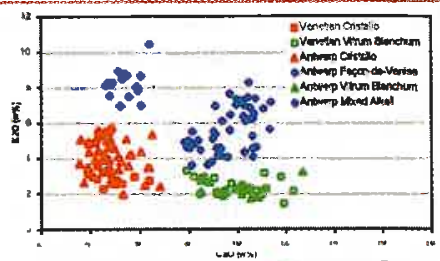

(b)

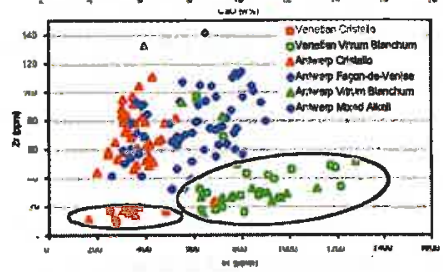

Fig 3: (a) $\mathrm{CaO} / \mathrm{K} 20$ (w\%) (EPMA) and (b) $\mathrm{Sr} / \mathrm{Zr}$ concentration scatter plots (ppm) ( $\mu$-SRXRF) of different types of 16-17th century glass objects excavated in Antwerp, Belgium. Circle fields in (b) denote the majority of the genuine Venetian 'Cristallo' and 'Vitrum Blanchum' objects.

tion of the sixteenth and seventeenth century Antwerp glass objects was compared to the major and trace composition of two genuine Venetian glass types, cristallo and vitrum blanchum. The analysis of the trace composition with $\mu$-SRXRF revealed that based on the impurity level of the used silica source, the zirconium content, we were able to make a distinction between local production and Venetian import.

[1] Vincze L., PhD Dissertation, University of Antwerp, 1995, 96

[2] Janssens K., De Raedt I., Schalm O., Veeckman J, Mikrochim. Acta [Suppl.] 15 (1998), 253-267

[3] Verità M., Rivista della Stazione Sperimentale del Vetro, 1, 1985, 17-29

[4] Bronk H., Mensch und Nuch Verlag, Berlin, Germany 1998, ISBN 3-933346-04-5
[5] Mortimer C., Trade and Discovery: The scientific study of Artefacts from Post-Medieval Europe and Beyond, occasional paper 109. Edited by D. R. Hook and D. R. M. Gaimster. British Museum Press 1995 